\title{
INFLUÊNCIA DA MASSA ESPECÍFICA SOBRE AS PROPRIEDADES MECÂNICAS DE PAINÉIS AGLOMERADOS
}

\author{
Setsuo Iwakiri*, Andréa Berriel Mercadante Stinghen**, Elenise Leocádia da Silveira**, \\ Esoline Helena Cavalli Zamarian***, José Guilherme Prata**, Marilene Bronoski** \\ *Eng. Florestal, Dr., Departamento de Engenharia e Tecnologia Florestal, UFPR - setsuo@ufpr.br \\ **M.Sc., Doutorando em Engenharia Florestal, UFPR - andreaberriel@onda.com.br - elenise.nunes@bol.com.br - \\ jgprata@gmail.com - marilene.bronoski@ig.com.br \\ ***Mestranda em Engenharia Florestal, UFPR - esoline@sanmarcoseguros.com.br \\ Recebido para publicação: 29/06/2007 - Aceito para publicação: 03/12/2007
}

\begin{abstract}
Resumo
Este trabalho teve como objetivos avaliar a influência da massa específica do painel aglomerado sobre suas propriedades mecânicas e verificar os níveis de correlações entre as variáveis estudadas. Foram produzidos painéis com massa específica nominal de $0,60,0,70,0,80$ e $0,90 \mathrm{~g} / \mathrm{cm}^{3}$, utilizando-se partículas de Pinus spp. coletadas na linha de produção industrial e resina uréia-formaldeído como aglutinante. Os valores médios obtidos para MOE e MOR em flexão estática, ligação interna e MOR em compressão paralela foram superiores para painéis com maiores massas específicas. $\mathrm{O}$ aumento na densidade do painel para valores em torno de $0,80 \mathrm{~g} / \mathrm{cm}^{3}$ resultou em incremento proporcionalmente superior no valor médio das propriedades mecânicas dos painéis. Esse resultado indica a viabilidade de fabricação de painéis aglomerados com massa específica acima de $0,80 \mathrm{~g} / \mathrm{cm}^{3}$ para aplicações específicas que requeiram alta resistência mecânica. Com exceção da ligação interna, a relação linear entre a massa específica e as variáveis dependentes - MOE e MOR em flexão estática e MOR em compressão paralela -, apresentou altos valores de coeficiente de determinação, indicando a possibilidade de predição dessas propriedades com base na massa específica dos painéis.

Palavras-chave: Painéis aglomerados; pinus; massa específica; propriedades mecânicas.
\end{abstract}

\begin{abstract}
Effects of the density on the mechanical properties of particleboard. The objective of this research was to evaluate the influences of the board density on the mechanical properties and to verify the correlations levels between the variables studied. They were manufactured panels with the nominal density of 0,$60 ; 0,70 ; 0,80$ and $0,90 \mathrm{~g} / \mathrm{cm}^{3}$, using particles of Pinus spp collected at the particleboad industry and urea-formaldehyde resin. The average values obtained for MOE and MOR in static bending, internal bond and compression parallel to grain become higher for as the board density increases. The increase in board density near of $0,80 \mathrm{~g} / \mathrm{cm}^{3}$, results in proportionally higher values of mechanical properties. This results indicate the possibility to manufacture the particleboard with density above to $0,80 \mathrm{~g} / \mathrm{cm}^{3}$ for specific applications that required high mechanical strength. With the exception of the internal bond, the linear relation between board density and dependents variables MOE and MOR in the static bending and MOR in the parallel compression to grain, showed high coefficient of determination indicating the possibility of predicting these properties basing on the board density.
\end{abstract}

Keywords: Particleboard; pinus; density; mechanical properties.

\section{INTRODUÇ̃̃O}

A produção de painéis aglomerados no Brasil teve início em 1966, quando entrou em operação a primeira linha de produção da empresa Placas do Paraná S.A., localizada em Curitiba (PR). Desde então, o número de unidades produtoras de painéis aglomerados tem aumentado de forma expressiva, com a produção atingindo o patamar de 2,0 milhões de $\mathrm{m}^{3} \mathrm{em} 2005$ (SBS, 2006).

$\mathrm{O}$ aglomerado é um painel produzido a partir de pequenas partículas de madeira impregnadas com resina uréia-formaldeído e consolidado através da aplicação de calor e pressão. É um produto 
amplamente empregado na fabricação de móveis, em função de suas características tecnológicas, custo competitivo e escala de produção (IWAKIRI et al., 2005). Atualmente, com o emprego da tecnologia de prensa contínua e melhor controle da granulometria das partículas, as características de painéis aglomerados passaram por melhorias significativas, principalmente no que se refere a sua densificação, resultando em melhor distribuição do gradiente vertical de densidade, com as faces mais compactadas e textura mais fina da superfície do painel.

A tecnologia empregada na produção de painéis aglomerados agrega uma série de vantagens, tais como: (i) eliminação do fator anisotrópico da madeira sólida; (ii) propriedades físicas e mecânicas mais homogêneas; (iii) eliminação de fatores redutores da resistência da madeira, como nós, inclinação da grã e lenho juvenil, entre outros; (iv) adequação das propriedades dos painéis através do controle dos parâmetros do processo; (v) menores exigências em termos de qualidade da madeira, como diâmetro da tora, forma do fuste, defeitos, etc; (vi) menor custo de produção, decorrente dos requisitos de qualidade da madeira e automação do processo produtivo (MALONEY, 1993; MOSLEMI, 1974).

A qualidade do painel aglomerado depende das interações entre inúmeras variáveis, como a espécie, a massa específica, o tipo e quantidade de resina, a geometria de partículas, a umidade do colchão e o ciclo de prensagem, entre outras (KELLY, 1977). De acordo com Maloney (1993), a razão de compactação, que é definida como a relação entre a massa específica do painel e a massa específica da madeira, é um parâmetro de grande importância na estabilidade dimensional e na resistência mecânica do painel aglomerado. Painéis com maior razão de compactação terão propriedades mecânicas superiores, mas com menor estabilidade dimensional. Segundo Moslemi (1974), essa relação pode ser modificada utilizando-se espécies de menor massa específica para a mesma massa específica do painel, ou, para a mesma espécie, aumentando-se a massa específica do painel.

Para painéis com maior massa específica, Marra (1992) recomenda o uso de maior quantidade de resina, tendo em vista a maior área superficial das partículas. Portanto, no procedimento para cálculo de materiais, deve-se levar em consideração a proporcionalidade entre a quantidade de partículas e a de resina para painéis de diferentes massas específicas. As propriedades mecânicas do painel aglomerado são diretamente proporcionais à sua massa específica. Na pesquisa realizada por Albuquerque (2002), foram verificados aumentos significativos das propriedades de ligação interna, do módulo de elasticidade e do módulo de ruptura em flexão estática, com aumento na massa específica do painel de 0,70 para 0,90 $\mathrm{g} / \mathrm{cm}^{3}$.

Este trabalho foi realizado com o objetivo de avaliar a influência da massa específica do painel sobre as propriedades mecânicas e, através da análise de regressão linear, verificar os níveis de correlações entre as variáveis estudadas.

\section{MATERIAIS E MÉTODOS}

Foram utilizadas nesta pesquisa partículas de Pinus spp. destinadas à camada interna de painéis aglomerados coletadas diretamente na linha de produção da empresa Placas do Paraná S.A. A resina empregada na colagem de partículas foi a uréia-formaldeído (UF), com teor de sólidos de 65\%, pH 8,7 e viscosidade de $278 \mathrm{cp}$.

A resina UF, em quantidade de $8 \%$ de sólidos, base peso seco das partículas, foi aplicada sobre as partículas numa encoladeira tipo tambor rotatório através de bico aspersor integrado ao compressor. $\mathrm{O}$ colchão de partículas foi formado numa caixa "vazada" com dimensões de 50 x $50 \mathrm{~cm}$, separadas por placas de alumínio. Após a pré-prensagem para melhor acomodação das partículas, o colchão foi prensado numa prensa a quente, com temperatura de $180^{\circ} \mathrm{C}$, pressão específica de $40 \mathrm{kgf} / \mathrm{cm}^{2}$ e tempo de prensagem de 7 minutos.

Foram produzidos doze painéis aglomerados com massa específica estimada de $0,60,0,70,0,80$ e $0,90 \mathrm{~g} / \mathrm{cm}^{3}$, identificados como tratamentos $\mathrm{T} 1, \mathrm{~T} 2, \mathrm{~T} 3 \mathrm{e} \mathrm{T}$, respectivamente, sendo realizadas três repetições por tratamento.

Após a prensagem, os painéis foram acondicionados em câmara de climatização à temperatura de $20 \pm 2{ }^{\circ} \mathrm{C}$ e umidade relativa de $65 \pm 3 \%$, até a sua estabilização. A seguir, foram retirados os corpos de prova para ensaios de flexão estática, compressão paralela e ligação interna. Os ensaios foram realizados com base nos procedimentos descritos na norma EN 310:1993 (flexão estática), ASTM D 
1037:1993 (compressão paralela) e EN 319:1993 (ligação interna). Para cada uma das propriedades, foram testados 16 corpos de prova por tratamento.

A análise estatística dos resultados foi realizada através de ANOVA e teste de Tukey, ao nível de probabilidade de $95 \%$. As relações lineares entre a variável independente (massa específica) e variáveis dependentes (MOE e MOR) em flexão estática, compressão paralela e ligação interna foram obtidas através da análise de regressão linear.

\section{RESULTADOS E DISCUSSÃO}

\section{Propriedades mecânicas dos painéis}

Os resultados obtidos para o módulo de elasticidade (MOE) e módulo de ruptura (MOR) em flexão estática estão apresentados na tabela 1.

Tabela 1. Valores médios de módulo de elasticidade (MOE) e de ruptura (MOR) em flexão estática.

Table 1. Average values of modulus of elasticity and rupture in static bending.

\begin{tabular}{lccc}
\hline Tratamento & $\begin{array}{c}\text { Massa específica } \\
\left(\mathbf{g} / \mathbf{c m}^{\mathbf{3}}\right)\end{array}$ & $\begin{array}{c}\text { MOE } \\
\left(\mathbf{k g f} / \mathbf{c m}^{\mathbf{2}}\right)\end{array}$ & $\begin{array}{c}\text { MOR } \\
\left(\mathbf{k g f} / \mathbf{c m}^{\mathbf{2}}\right)\end{array}$ \\
\hline $\mathrm{T} 1$ & 0,57 & $12.084 \mathrm{a}$ & $92 \mathrm{a}$ \\
$\mathrm{T} 2$ & 0,64 & $15.572 \mathrm{~b}$ & $124 \mathrm{~b}$ \\
$\mathrm{~T} 3$ & 0,70 & $16.736 \mathrm{~b}$ & $143 \mathrm{~b}$ \\
$\mathrm{~T} 4$ & 0,78 & $22.446 \mathrm{c}$ & $188 \mathrm{c}$ \\
\hline
\end{tabular}

Os resultados da tabela 1 demonstram que tanto para o MOE quanto para o MOR o aumento na massa específica do painel resultou em aumento nos valores médios dessas propriedades. Entre os tratamentos T1, com menor massa específica, tratamentos T2 e T3, com massa específica intermediária, e tratamento T4, com maior massa específica, foram constatadas diferenças estatisticamente significativas ao nível de probabilidade de $95 \%$. Levando-se em consideração esses três patamares de aumento nos valores médios de MOE e MOR, pode-se constatar que há um incremento proporcionalmente maior dessas propriedades com o aumento da massa específica dos painéis de $0,57 \mathrm{~g} / \mathrm{cm}^{3}$ (T1) para $0,64 \mathrm{~g} / \mathrm{cm}^{3}$ (T2), e entre o tratamento T3, com massa específica de $0,70 \mathrm{~g} / \mathrm{cm}^{3}$, e o tratamento T4, com massa específica de $0,78 \mathrm{~g} / \mathrm{cm}^{3}$. Entre os tratamentos T2 e T3, cujo aumento da massa específica foi de 0,64 $\mathrm{g} / \mathrm{cm}^{3}$ para $0,70 \mathrm{~g} / \mathrm{cm}^{3}$, os valores médios de MOE e MOR foram estatisticamente iguais. Esses resultados apontam para a possibilidade de fabricação de painéis aglomerados de maior densificação para aplicações específicas que requeiram maior resistência mecânica. Os valores médios de MOE e MOR obtidos neste trabalho foram superiores aos valores encontrados por Albuquerque (2002), para painéis aglomerados com massa específica de 0,70 e $0,90 \mathrm{~g} / \mathrm{cm}^{3}$, cujos valores médios foram respectivamente de 10.798 $\mathrm{kgf} / \mathrm{cm}^{2}$ e $13.111 \mathrm{kgf} / \mathrm{cm}^{2}$ para MOE e de $94 \mathrm{kgf} / \mathrm{cm}^{2}$ e $139 \mathrm{kgf} / \mathrm{cm}^{2}$ para MOR.

Na tabela 2 estão apresentados os resultados obtidos para a ligação interna (LI) e a compressão paralela $(\mathrm{CP})$.

Tabela 2. Valores médios de compressão paralela e ligação interna.

Table 2. Average values of compression paralell to the grain and internal bond.

\begin{tabular}{lcccc}
\hline Tratamento & $\begin{array}{c}\text { Massa específica } \\
\left(\mathbf{g} / \mathbf{c m}^{\mathbf{3}}\right)\end{array}$ & $\begin{array}{c}\text { Ligação interna } \\
\left(\mathbf{k g f} / \mathbf{c m}^{2}\right)\end{array}$ & $\begin{array}{c}\text { Massa específica } \\
\left(\mathbf{g} / \mathbf{c m}^{\mathbf{3}}\right)\end{array}$ & $\begin{array}{c}\text { MOR - compressão } \\
\text { paralela }\left(\mathbf{k g f} / \mathbf{c m}^{\mathbf{2}}\right)\end{array}$ \\
\hline $\mathrm{T} 1$ & 061 & $5,80 \mathrm{a}$ & 0,60 & $66 \mathrm{a}$ \\
$\mathrm{T} 2$ & 0,68 & $7,42 \mathrm{~b}$ & 0,68 & $102 \mathrm{~b}$ \\
$\mathrm{~T} 3$ & 0,77 & $6,78 \mathrm{ab}$ & 0,74 & $124 \mathrm{c}$ \\
$\mathrm{T} 4$ & 0,85 & $7,79 \mathrm{~b}$ & 0,83 & $159 \mathrm{~d}$ \\
\hline
\end{tabular}

Os valores médios da ligação interna apresentados na tabela 2 indicam que apenas os tratamentos T3 e T4, com maior massa específica dos painéis, apresentaram resultados estatisticamente superiores em comparação aos painéis do tratamento T1, com menor massa específica. Para o tratamento T3, não foram constatadas diferenças estatisticamente significativas em relação aos demais tratamentos, embora em termos de médias absolutas o valor médio obtido seja superior ao tratamento T1, com menor massa 
específica dos painéis. A redução do valor médio de ligação interna observada no tratamento T3 em relação ao tratamento T2, com menor massa específica, influenciou significativamente na relação linear entre essa propriedade e a massa específica dos painéis, o que pode ser constatado pelo baixo valor do coeficiente de determinação $\left(\mathrm{R}^{2}=0,28\right)$ apresentado na tabela 3. Todos os valores médios de ligação interna obtidos neste trabalho atendem ao requisito da Norma Européia EN 312 (1993), cujo valor mínimo é de $3,57 \mathrm{kgf} / \mathrm{cm}^{2}(0,35 \mathrm{MPa})$.

Os resultados obtidos para a compressão paralela indicam forte influência da massa específica do painel sobre essa propriedade. Para os quatro diferentes níveis de massa específica do painel, foram constatadas diferenças estatisticamente significativas entre os valores médios de compressão paralela, o que contribuiu significativamente para a obtenção de alto coeficiente de determinação $\left(\mathrm{R}^{2}=0,96\right)$ na relação linear entre essas propriedades, conforme apresentado na tabela 3. Cabe ressaltar também que, com o aumento na densidade do painel para valor acima de $0,80 \mathrm{~g} / \mathrm{cm}^{3}$, constatou-se um incremento proporcionalmente superior no valor médio de MOR em compressão paralela. Esse resultado indica as vantagens decorrentes da fabricação de painéis aglomerados com densidade acima de $0,80 \mathrm{~g} / \mathrm{cm}^{3}$ visando aplicações específicas que requeiram maior resistência mecânica. Vale lembrar que a compressão paralela é um dos ensaios mecânicos que melhor reflete a resistência real de produtos de madeira.

Para todas as propriedades mecânicas avaliadas, foram constatadas relações diretamente proporcionais com a massa específica do painel. A influência da massa específica sobre as propriedades mecânicas dos painéis aglomerados pode ser atribuída à razão de compactação das partículas que constituem o colchão. De acordo com Maloney (1993), quanto maior a massa específica do painel, maior será a quantidade de partículas e, conseqüentemente, maior será a compactação do material durante a prensagem e conformação do painel. Neste estudo, foi constatado que painéis com massa específica acima de $0,80 \mathrm{~g} / \mathrm{cm}^{3}$ resultam em aumentos expressivos de suas propriedades mecânicas.

\section{Relações lineares entre as variáveis estudadas}

Os resultados das análises de regressões lineares entre as massas específicas e as propriedades mecânicas dos painéis aglomerados estão apresentados na tabela 3 e nas figuras 1, 2, 3 e 4 .

Tabela 3. Equações de regressão linear entre as variáveis estudadas e a massa específica a 95\% de probabilidade.

Table 3. Equations of linear regression between studied variables and board density at probability of $95 \%$.

\begin{tabular}{lrrr}
\hline Equação de regressão & Syx & $\mathbf{F}$ & $\mathbf{R}^{\mathbf{2}}$ \\
\hline $\mathrm{MOE}=-15971,3+48726,6 \times \mathrm{ME}$ & 1441,17 & 571,92 & 0,90 \\
$\mathrm{MOR}=-138,82+408,186 \times \mathrm{ME}$ & 15,77 & 334,84 & 0,84 \\
$\mathrm{CP}=-184,345+417,001 \times \mathrm{ME}$ & 8,37 & 1353,31 & 0,96 \\
$\mathrm{LI}=1,22109+7,84852 \times \mathrm{ME}$ & 1,29 & 23,97 & 0,28 \\
\hline
\end{tabular}

$\mathrm{ME}=$ massa específica do painel; $\mathrm{MOE}=$ módulo de elasticidade em flexão estática; $\mathrm{MOR}=$ módulo de ruptura em flexão estática; $\mathrm{CP}=$ compressão paralela; $\mathrm{LI}=$ ligação interna; Syx: erro padrão de estimativa; $\mathrm{r}=$ coeficiente de correlação; $\mathrm{R}^{2}=$ coeficiente de determinação.

De acordo com as figuras 1, 2 e 3, as relações lineares entre a massa específica do painel e as propriedades mecânicas de MOE e MOR em flexão estática e MOR em compressão paralela apresentaram alto coeficiente de determinação $\left(\mathrm{R}^{2}\right)$ nos intervalos estudados, com valores de 0,90, 084 e 0,96 , respectivamente. Os valores de $\mathrm{R}^{2}$ encontrados permitem afirmar que as equações de regressão geradas nas análises realizadas possibilitam a predição do MOE e MOR em flexão estática e MOR em compressão paralela com base na massa específica dos painéis, ao nível de probabilidade de 99\%. Por outro lado, a relação linear entre a massa específica do painel e a ligação interna apresentada na figura 4. indica um baixo coeficiente de determinação $\left(\mathrm{R}^{2}\right)$ no intervalo estudado, com valor de 0,28. Esse resultado indica a impossibilidade de predição da ligação interna com base na massa específica dos painéis, ao nível de probabilidade de $99 \%$. 


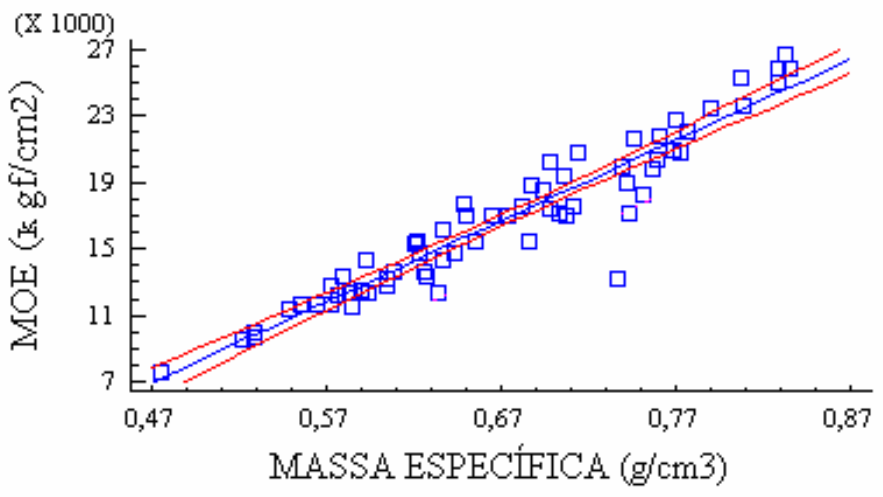

Figura 1. Regressão linear entre MOE de flexão estática e massa específica do painel. Figure 1. Linear regression between MOE in static bending and board density.

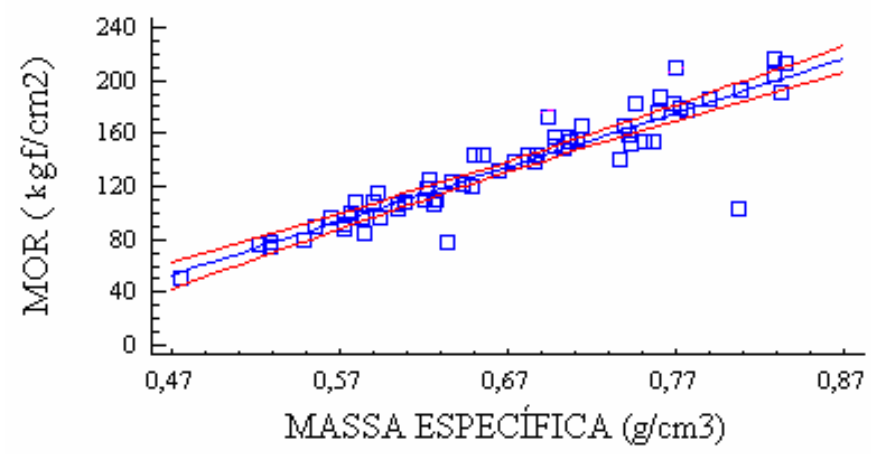

Figura 2. Regressão linear entre MOR de flexão estática e massa específica do painel. Figure 2. Linear regression between MOR in static bending and board density.

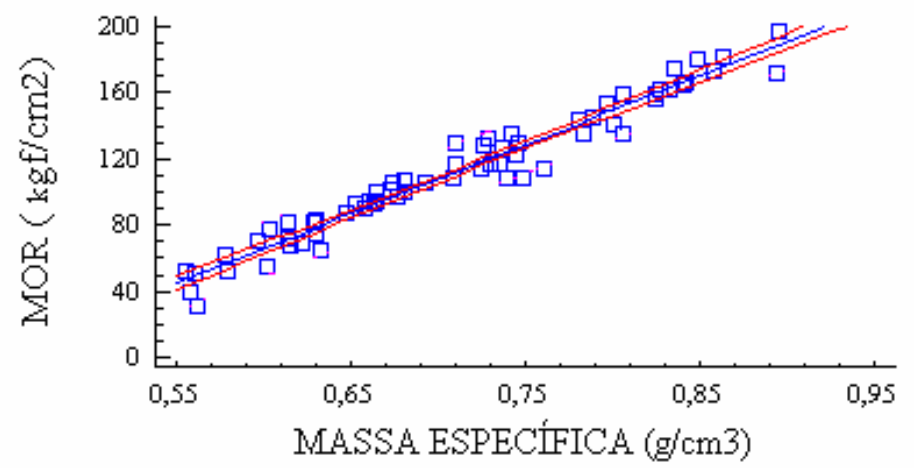

Figura 3. Regressão linear entre compressão paralela e massa específica do painel.

Figure 3. Linear regression between compression parallel of the grain and board density. 


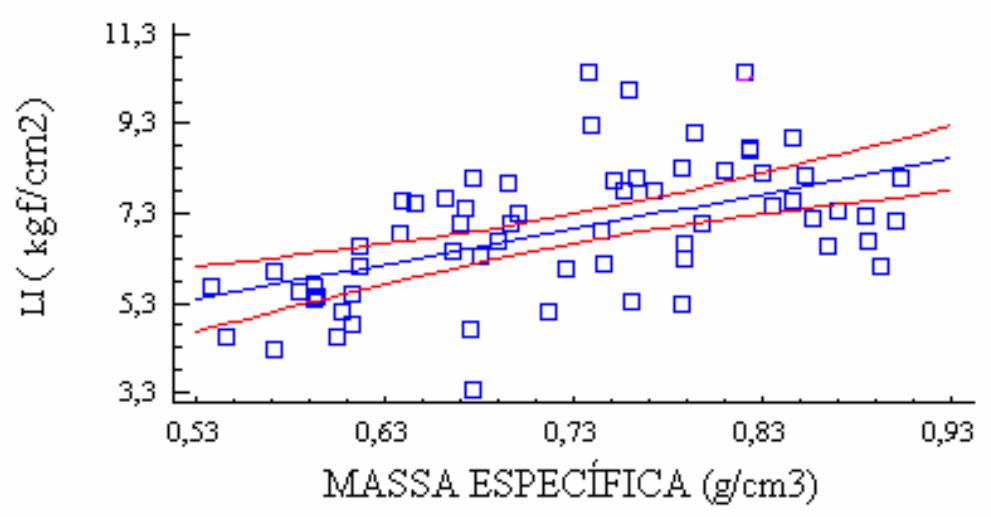

Figura 4. Regressão linear entre ligação interna e massa específica do painel. Figure 4. Linear regression between internal bond and board density.

\section{CONCLUSÕES}

Com base nos resultados obtidos, as seguintes conclusões podem ser apresentadas:

- Os painéis aglomerados produzidos com maiores massas específicas apresentaram valores superiores de módulo de elasticidade e ruptura em flexão estática, ligação interna e compressão paralela.

- O aumento na densidade do painel na faixa de 0,70 para $0,80 \mathrm{~g} / \mathrm{cm}^{3}$ resultou em incrementos proporcionalmente superiores nos valores médios de módulo de elasticidade e módulo de ruptura em flexão estática.

- Os resultados obtidos indicam a possibilidade de fabricação de painéis aglomerados com massa específica acima de $0,80 \mathrm{~g} / \mathrm{cm}^{3}$ para aplicações específicas que requeiram alta resistência mecânica.

- As relações lineares entre a massa específica e as propriedades mecânicas de MOE e MOR em flexão estática e compressão paralela apresentaram altos valores de coeficiente de determinação, indicando a possibilidade de predição dessas propriedades com base na massa específica dos painéis aglomerados.

\section{REFERÊNCIAS}

ALBUQUERQUE, C. E. C. Interações de variáveis no ciclo de prensagem de aglomerados. $150 \mathrm{f}$. Tese (Doutorado em Ciências Florestais) - Setor de Ciências Agrárias, Universidade Federal do Paraná. Curitiba. 2002.

AMERICAN SOCIETY FOR TESTING MATERIALS. ASTM D-1037: standard methods of evaluating the properties of wood-base fiber and particle materials. In: Philladelphia, 1993. . Annual Book of ASTM standards.

EUROPEAN COMMITTEE FOR STANDARDIZATION. EN 310: wood based panels - determination of modulus of elasticity in bending and of bending strength. Brussels, 1993.

EUROPEAN COMMITTEE FOR STANDARDIZATION. EN 319: particleboards and fiberboards Determination of tensile strength perpendicular to the plane of the board. Brussels, 1993.

IWAKIRI, S.; ALBUQUERQUE, C. E. C.; MENDES, L. M.; LATORRACA, J. V. F. Painéis de madeira aglomerada. In: IWAKIRI, S. (Org.). Painéis de madeira reconstituída. Curitiba: FUPEF, 2005. p. 137182.

KELLY, M. W. A critical literature review of relationship between processing parameters and physical properties of particleboard. Madison: U.S. Forest Products Lababoratory, 1977. 66 p. (General Technical Report, FPL-10). 
MALONEY, T. M. Modern particleboard and dry-process fiberboard manufacturing. 2. ed. San Francisco: M. Freeman, 1993. 689 p.

MARRA, A. A. Technology of wood bonding: principles in practice. New York: Van Nostrand Reinhold. 1992. 453 p.

MOSLEMI, A. A. Particleboard: vol I: materils. London: Southern University Press, 1974. 244 p.

SOCIEDADE BRASILEIRA DE SILVICULTURA. Fatos e números do Brasil Florestal - 2006.

Disponível em: <http://www.sbs.org.br>. Acesso em: 15/07/2007. 